$\underline{\beta}=-m$

\title{
Pharmacological evaluation of oxazine sulfonamide derivatives for anti-inflammatory, analgesic and antipyretic activities
}

\author{
ShailajaAnde $^{1 *}$, Anjum $^{2}$, KhadarShaik ${ }^{2}$, NisaFirdouse $^{2}$, RavindracharyDharmoji $^{2}$, SowmyaRaginiYelwarthi $^{2}$ \\ ${ }^{1}$ M.Pharm, Malla Reddy Institute of Pharmaceutical sciences, Secundrabad \\ ${ }^{2}$ B.Pharm, GyanaJyothi College of Pharmacy, Uppal, Hyderabad-98, Telangana, India \\ *Corresponding author E-mail:genuine.shaila@gmail.com
}

\begin{abstract}
Oxazine heterocyclic compound containing one oxygen and one nitrogen atom. They possess different pharmacological activities like anticancer, anti-ulcer, antifungal, antithrombotic, antihypertensive, analgesic, anti-inflammatory etc. Oxazine Sulphonamide derivatives were investigated for anti-inflammatory, analgesic and anti-pyretic activities. It was evaluated for anti-inflammatory activity by carrageenan induced paw edema in rats using Plethysmometer and it also shows significant analgesic effect in male Albino mice. Antipyretic activity was also investigated.
\end{abstract}

Keywords: Analgesic; Ant-Pyretic; Anti-Inflammatory; Oxazine Sulphonamide Derivatives; Yeast; Carrageenan; Plethysmometer.

\section{Introduction}

Oxazinea heterocyclic compound containing one oxygen and one nitrogen atom. Many isomers exists depending on the relative position of the heteroatoms and relative position of the double bonds. The examples of Oxazine derivatives include MorphineTetrahydro-1,4-Oxazine, ifosfamide a nitrogen mustard alkylating agent used in the treatment of cancer.<smiles>C1COCCN1</smiles>

Morphine-

Tetrahydro1,4-Oxazine

Benzoxazine is a molecule where an oxazine ring is attached to a benzene ring. There are several benzoxazines depending on the position of oxygen and nitrogen heteroatoms. Benzoxazine is the compound with double bond in the Oxazine ring. They posseses different pharmacological activities like anticancer, anti ulcer, antifungal, antithrombotic, antihypertensive, analgesic, anti inflammatory etc.

\section{Materials and methods}

\subsection{Materials}

For analgesic activity, pentazocine is used as standard drug and the animals were treated with Oxazine as test compound. For anti pyretic activity, yeast is used to induce pyrexia and standard drug used was paracetamol. The animals are also treated with test drug.
For anti inflammatory activity, carragenan is used for inducing paw edema, Oxazine sulphonamide derivatives used as test and diclofenac is used as standard drug.

\subsection{Methods}

Analgesic activity was carried out with Hot Plate Latency Test using Eddy's Hot plate method and Antipyretic activity was done by using Yeast inducing Hyper pyrexia method. Anti inflammatory activity is measured by using Plethysmometer by carrageenaninduced hind paw edema in rats.

\subsection{Experimental animals}

Healthy adult male Albino mice (25-35g) were selected for analgesic activity. They were maintained under proper conditions. Healthy adult male Wistar rats $(150-250 \mathrm{~g})$ were used for anti pyretic activity. They were fed with water and standard pellets and maintained under proper environment. For anti inflammatory activity healthy adult male rats $(150-250 \mathrm{~g})$ were used. They were housed in polypropylene cages and maintained under suitable nutritional and environmental conditions. Animals were fed with water and pellets.

\section{Results and Discussions}

The study showed that the Oxazine sulphonamide derivatives has potent anti-inflammatory and analgesic activities but not antipyretic action. This was confirmed by the observations from all the models of anti-inflammatory, antipyretic and analgesic activity used in this study.

\subsection{Results}

\subsubsection{Acute toxicity study}


The acute oral toxicity procedure was followed by using OECD 423 guidelines.

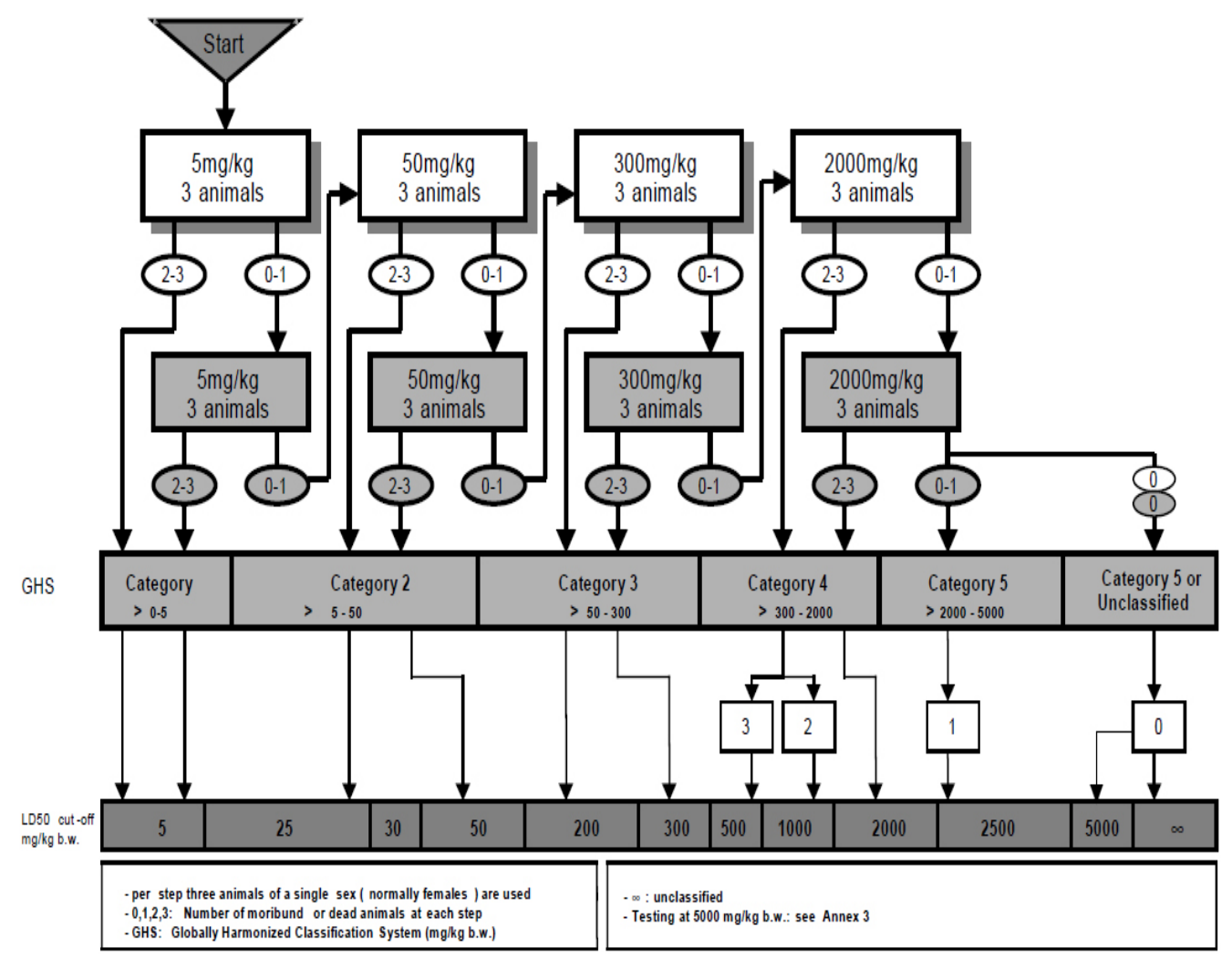

Fig.1: Acute Toxicity Design

\subsubsection{Screening of analgesic activity}

\section{Hot plate latency test}

The hot plate latency assay was carried out according to the method of Eddy et al.,1950.the rats used for this study were divided into 7 groups, 5groups received the test compound 1,test compound 2 , test compound 3 ,test compound 4 ,test compound 5, while the remaining two groups was std and control. The test drugs, control and standard were administered orally to the animals after $12 \mathrm{hrs}$ of fasting. The animals were each placed on a hot plate maintained at $55^{\circ} \mathrm{C}, 30 \mathrm{~min}$ after administration of test compounds, standard and saline. The time taken for the rats to respond to the thermal stimulus (usually by jumping) was noted as the latency (in second). The mean of the latency for each group was determined. The effects of the test drugs, standard and saline were also determined after $0,30,60,120,180 \mathrm{~min}$ of administration to rats.

Table 1: Animals and Treatment for Analgesic Activity

\begin{tabular}{|c|c|}
\hline $\operatorname{Group}(n=6)$ & Treatment \\
\hline 1 & Water(control) \\
\hline 2 & Std drug (pentazocine $5 \mathrm{mg} / \mathrm{kg}$ ) \\
\hline 3 & *Bvk.ks.01.14(10mg/kg) \\
\hline 4 & *Bvk.ks.01.16(10mg/kg) \\
\hline 5 & *Bvk.ks.01.17(10mg/kg) \\
\hline 6 & *Bvk.ks.01.18(10mg/kg) \\
\hline 7 & *Bvk.ks.01.20(10mg/kg) \\
\hline
\end{tabular}

Table 2: Analgesic Responses of Drugs with Time in Analgesic Activity

\begin{tabular}{|c|c|c|c|c|c|}
\hline Group and dose & 0min & $30 \mathrm{~min}$ & $60 \mathrm{~min}$ & $120 \mathrm{~min}$ & $180 \mathrm{~min}$ \\
\hline Control & $7+1.7$ & $6+0.8$ & $6+2.0$ & $7+0.8$ & $6.8+1.4$ \\
\hline $\operatorname{Std}(5 \mathrm{mg} / \mathrm{kg})$ & $\begin{array}{l}5.6+1 . \\
8\end{array}$ & $\begin{array}{l}10.8+1 . \\
4 * *\end{array}$ & $\begin{array}{l}12.6+2 . \\
1 * *\end{array}$ & $\begin{array}{l}15.3+2 . \\
8 * *\end{array}$ & $\begin{array}{l}17.5+2 . \\
1 * *\end{array}$ \\
\hline $\begin{array}{l}\text { *Bvk.ks.01.14(10 } \\
\mathrm{mg} / \mathrm{kg})\end{array}$ & $7+1.2$ & $\begin{array}{l}10.5+1 . \\
0 * *\end{array}$ & $\begin{array}{l}15+2.1 * \\
*\end{array}$ & $\begin{array}{l}16.8+1 . \\
1 * *\end{array}$ & $\begin{array}{l}17.3+0 . \\
8 * *\end{array}$ \\
\hline $\begin{array}{l}\text { *Bvk.ks.01.16(10 } \\
\mathrm{mg} / \mathrm{kg})\end{array}$ & $\begin{array}{l}7.16+ \\
0.7\end{array}$ & $\begin{array}{l}9.6+1.3 \\
* *\end{array}$ & $\begin{array}{l}13.3+1 \\
9 * *\end{array}$ & $\begin{array}{l}15.6+1 \\
6^{* *}\end{array}$ & $\begin{array}{l}16.8+1 . \\
1^{* *}\end{array}$ \\
\hline $\begin{array}{l}\text { * Bvk.ks.01.17(10 } \\
\mathrm{mg} / \mathrm{kg})\end{array}$ & $\begin{array}{l}7.5+1 . \\
0\end{array}$ & $\begin{array}{l}11.5+1 . \\
5^{* *}\end{array}$ & $\begin{array}{l}14.6+1 . \\
5^{* *}\end{array}$ & $\begin{array}{l}17.6+1 . \\
3 * *\end{array}$ & $\begin{array}{l}16.6+1 . \\
2^{* * *}\end{array}$ \\
\hline $\begin{array}{l}\text { *Bvk.ks.01.18(10 } \\
\mathrm{mg} / \mathrm{kg})\end{array}$ & $\begin{array}{l}7.66+ \\
1.0\end{array}$ & $\begin{array}{l}11.5+1 . \\
8 * *\end{array}$ & $\begin{array}{l}14.8+2 . \\
1 * *\end{array}$ & $\begin{array}{l}16.6+2 . \\
0^{* *}\end{array}$ & $\begin{array}{l}15.3+0 . \\
8^{* *}\end{array}$ \\
\hline $\begin{array}{l}\text { *Bvk.ks.01.20(10 } \\
\mathrm{mg} / \mathrm{kg})\end{array}$ & $\begin{array}{l}7.16+ \\
0.7\end{array}$ & $\begin{array}{l}9.5+0.8 \\
* *\end{array}$ & $\begin{array}{l}14.1+1 . \\
4 * *\end{array}$ & $\begin{array}{l}16+0.8 * \\
*\end{array}$ & $\begin{array}{l}16.5+1 . \\
6 * *\end{array}$ \\
\hline
\end{tabular}

Values are expressed in mean+SEM, significant $\mathrm{P}^{* *}<0.01 ; \mathrm{P}^{*}>0.05$ compared to control.

Data 1

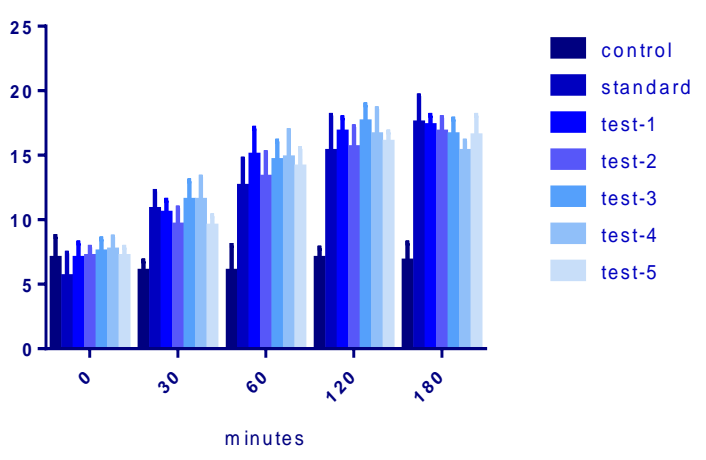

$\mathrm{X}$ axis-minutes

$\mathrm{Y}$ axis-time (sec)

Fig. 2: Graph Representing the Analgesic Activity Results 


\subsubsection{Screening of anti pyretic activity}

\section{Yeast inducing hyperpyrexia method}

The antipyretic activities of test compounds were evaluated by using digital thermometer. The antipyretic activity was screened by using yeast induced hyperpyrexia method. Rats were selected and divided into seven groups each having six animals. The animals were administered control,std and test drugs as shown in the table. They were maintained at standard at constant temperature of 97 to $98{ }^{0}$ Ffor 24 hours before pyrexia was induced. Experimental pyrexia induced by $15 \%$ suspension of Brewer's yeast in $0.9 \%$ saline solution by giving $10 \mathrm{ml} / \mathrm{kg}$. The rectal temperature before and after treatment was recorded with the help of digital clinical thermometer atevery hour up to 3 hours and was compare with control . Before yeast injection the rectal temperature was recorded. The yeast injection was given to all the groups. Immediately after yeast injection increase in the rectal temperature is recorded after $30 \mathrm{~min}$. The control, standardand test compounds1-5 were administered orally.The rectal temperature was recorded using digital thermometer prod was inserted 3-4 $\mathrm{cm}$ deep into the rectum.

Table 3: Animals and Treatment for Pyretic Activity

\begin{tabular}{ll}
\hline Group $(\mathrm{n}=6)$ & Treatment \\
\hline $1($ control $)$ & Control(water) \\
2(std) & Paracetamol $10 \mathrm{mg} / \mathrm{kg}$ \\
3 Test compound & $*$ Bvk.ks.01.14(10mg $/ \mathrm{kg})$ \\
4 Test compound & $*$ Bvk.ks.01.16(10mg/kg) \\
5 Test compound & *Bvk.ks.01.17(10mg $/ \mathrm{kg})$ \\
6 Test compound & $*$ Bvk.ks.01.18(10mg $/ \mathrm{kg})$ \\
7 Test compound & $*$ Bvk.ks.01.20 $(10 \mathrm{mg} / \mathrm{kg})$ \\
\hline
\end{tabular}

Table 4: Anti-pyretic Responses of Drugs with Time in Anti-pyretic Activity

\begin{tabular}{|c|c|c|c|c|c|}
\hline \multirow[b]{2}{*}{ Group and dose } & \multicolumn{5}{|c|}{ Rectal temperature in ${ }^{\circ} \mathrm{F}$ at different time intervals } \\
\hline & $\begin{array}{l}30 \\
\min \end{array}$ & $60 \mathrm{~min}$ & $90 \mathrm{~min}$ & $120 \mathrm{~min}$ & $180 \mathrm{~min}$ \\
\hline Control & $\begin{array}{l}99.4+ \\
0.6\end{array}$ & $\begin{array}{l}99.4+0 . \\
4\end{array}$ & $99+0.4$ & $99.2+0.6$ & $\begin{array}{l}99.2+0 . \\
5\end{array}$ \\
\hline Std(Paracetamol & $99.1+$ & $98.6+0$ & $98.4+0$. & $97.8+0.2$ & $97.5+0$. \\
\hline ) & 0.6 & 4 & 3 & & $1 * *$ \\
\hline *Bvk.ks.01.14(1 & 100.5 & $101.2+1$ & $100.8+1$ & $101.8+1$ & $100.9+0$ \\
\hline $0 \mathrm{mg} / \mathrm{kg})$ & +1.2 & $.2 * *$ & $.1 *$ & $* *$ & $.4 * *$ \\
\hline *Bvk.ks.01.16(1 & $99.8+$ & $101.2+0$ & $102+0.4$ & $101.5+0$ & $101.8+0$ \\
\hline $0 \mathrm{mg} / \mathrm{kg})$ & 1.0 & $.6 * *$ & $* *$ & $3 * *$ & $.4 * *$ \\
\hline *Bvk.ks.01.17(1 & 100.9 & $100.5+1$ & $100.9+1$ & $101+0.8$ & $101.8+0$ \\
\hline $0 \mathrm{mg} / \mathrm{kg})$ & +1.1 & .27 & $.2 *$ & $* *$ & $.4 * *$ \\
\hline *Bvk.ks.01.18(1 & 100.3 & $100.5+1$ & $101.3+1$ & $100.5+0$ & $101.2+1$ \\
\hline $0 \mathrm{mg} / \mathrm{kg})$ & +1.2 & .2 & $.1 * *$ & $9 * *$ & $.0 * *$ \\
\hline *Bvk.ks.01.20(1 & $99.9+$ & $100.7+0$ & $101.4+0$ & $101.3+0$ & $101.4+0$ \\
\hline $0 \mathrm{mg} / \mathrm{kg})$ & 0.9 & .65 & $.2 * *$ & $68 * *$ & $.6 * *$ \\
\hline
\end{tabular}

Values are expressed in Mean+SEM, $\mathrm{P}^{*}<0.05 \mathrm{P}^{* *}<0.01$ significant

Data 1

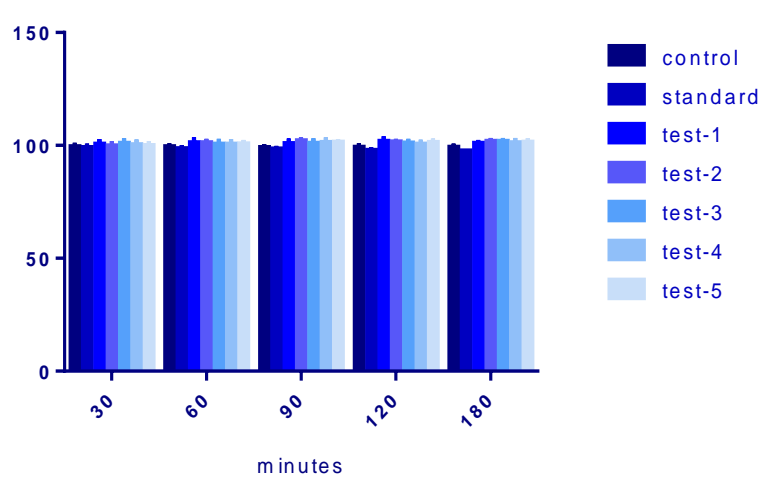

$\mathrm{X}$-axis $=$ minutes

$\mathrm{Y}$ axis $=$ temperature in ${ }^{\circ} \mathrm{C}$

Figure 3: Graph Representing the Anti-pyretic Activity Results

\subsubsection{Screening of anti inflammatory activity}

\section{Carrageenan-induced hind paw edema in rats}

The acute anti inflammatory effect was evaluated by carrageenan induced rat paw edema (Prabhakar et al.,2006). Edema was induced by injecting carrageenan $(1 \% \mathrm{w} / \mathrm{v}, 0.1 \mathrm{ml})$ in the right hind paw of rats. The test compounds $1-5$, diclofenac $(10 \mathrm{mg} / \mathrm{kg})$ and vehicle was administered orally one hour befor injection of carrageenan. Paw volume was measured with digital plethysmometer after 30, 60,120,180 min of injection.

Percentage increase $=\mathrm{A}-\mathrm{B} / \mathrm{A} * 100$

a) Paw volume of at different time intervals after injection.

b) Paw volume before injection.

Table 5: Animals and Treatment for Anti-inflammatory Activity

\begin{tabular}{ll}
\hline Groups $(\mathrm{n}=6)$ & Treatment \\
\hline $1($ control $)$ & water+carrageenan \\
$2(\mathrm{std})$ & Diclofenac $10 \mathrm{mg} / \mathrm{kg}$ \\
3 & *Bvk.ks.01.14(10mg/kg) \\
4 & *Bvk.ks.01.16(10mg/kg) \\
5 & *Bvk.ks.01.17(10mg/kg) \\
6 & *Bvk.ks.01.18(10mg/kg) \\
7 & *Bvk.ks.01.20 $(10 \mathrm{mg} / \mathrm{kg})$ \\
\hline
\end{tabular}

Table 6: Anti-inflammatory Responses of Drugs with Time in Antiinflammatory Activity

\begin{tabular}{|c|c|c|c|c|c|}
\hline Group \&dose & $0 \mathrm{~min}$ & $30 \mathrm{~min}$ & $60 \mathrm{~min}$ & $120 \mathrm{~min}$ & $180 \mathrm{~min}$ \\
\hline Control & $\begin{array}{l}0.71+0 . \\
07\end{array}$ & $\begin{array}{l}0.83+0 . \\
08\end{array}$ & $\begin{array}{l}0.86+0 . \\
05\end{array}$ & $\begin{array}{l}1.03+0 \\
13\end{array}$ & $\begin{array}{l}1.35+0 \\
25\end{array}$ \\
\hline Std $(10 \mathrm{mg} / \mathrm{kg})$ & $\begin{array}{l}0.72+0 . \\
09^{*}\end{array}$ & $\begin{array}{l}0.21+0 \\
03 * *\end{array}$ & $\begin{array}{l}0.23+0 \\
04 * *\end{array}$ & $\begin{array}{l}0.22+0 \\
05 * *\end{array}$ & $\begin{array}{l}0.30+0 . \\
05^{* *}\end{array}$ \\
\hline *Bvk.ks.01.14(1 & $0.85+0$ & $0.5+0.0$ & $0.37+0$ & $0.34+0$ & $0.28+0$ \\
\hline $0 \mathrm{mg} / \mathrm{kg})$ & $05^{*}$ & $6^{* *}$ & $04 * *$ & $04 * *$ & $07 * *$ \\
\hline *Bvk.ks.01.16(1 & $0.8+0.0$ & $0.42+0$ & $0.38+0$ & $0.31+0$ & $0.26+0$ \\
\hline 0mg/kg) & 8 & $04 * *$ & $04 * *$ & $04 * *$ & $05^{* *}$ \\
\hline *Bvk.ks.01.17(1 & $0.8+0.0$ & $0.53+0$ & $0.43+0$ & $0.35+0$ & $0.28+0$ \\
\hline $0 \mathrm{mg} / \mathrm{kg})$ & 8 & $05^{* *}$ & $07 * *$ & $05^{* *}$ & $04 * *$ \\
\hline $\begin{array}{l}\text { *Bvk.ks.01.18(1 } \\
0 \mathrm{mg} / \mathrm{kg})\end{array}$ & $\begin{array}{l}0.75+0 \\
10\end{array}$ & $\begin{array}{l}0.48+0 \\
07 * *\end{array}$ & $\begin{array}{l}0.41+0 \\
07 * *\end{array}$ & $\begin{array}{l}0.38+0 . \\
07 * *\end{array}$ & $\begin{array}{l}0.31+0 \\
04 * *\end{array}$ \\
\hline *Bvk.ks.01.20(1 & $0.78+0$ & $0.56+0$ & $0.48+0$ & $0.41+0$ & $0.35+0$ \\
\hline $0 \mathrm{mg} / \mathrm{kg})$ & 09 & $16^{* * *}$ & $04 * *$ & $04 * *$ & $08 * *$ \\
\hline
\end{tabular}

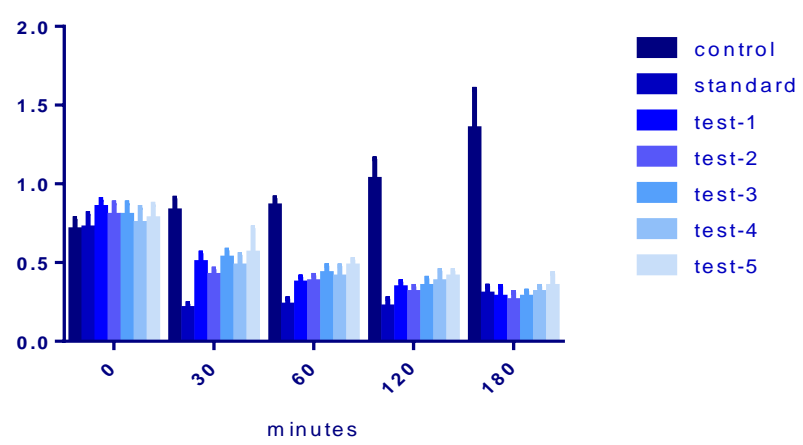

$\mathrm{X}$-axis $=$ minutes

$\mathrm{Y}$-axis = paw volume

Figure 4: Graph Representing the Anti-inflammatory Activity Results

\subsection{Discussions}

\subsubsection{Anti-pyretic activity}

Fever is provoked by many exogenous substances in animal models, including bacteria, endotoxins and microbe infection. Exogenous pyrogen induced the production of pro-inflammatory cyto- 
kinins such as IL-1 $\beta$, IL-6, interferons and Tumour Necrosis Factor which enter hypothalamic circulation and stimulate the release of local PG'S thereby resetting the hypothalamic thermal set point. In this study, Oxazine sulphonamide derivatives have not showed antipyretic action.

\subsubsection{Analgesic activity}

The test elucidates central and peripheral activities of nociception. The response pattern has two distinct periods of paw licking activity, an early response (0-5min after injection ) and a late response (20-30mins after injection). Early phase is due to direct effect of drug on nociceptors (anti-inflammatory). The late phase response is develop due to an inflammatory response and release analgesic mediators which reflect inflammatory pain. Experimental results have indicated that substance $\mathrm{p}$ and Bradykinin participate in early phase and histamine, serotonin and PG's are involved in late phase. The study showed analgesic activity of Oxazine sulphonamide derivativeson both phases of this test. Suggestingboth direct analgesic effect on the nociceptor and an inhibition of inflammatory pain. Thus these mode of actions probably involves the synthesis and release ofPG's or other pain mediators.

\subsubsection{Anti-inflammatory activity}

Inflammation occurs in three distinct phases-Acute, Sub-acute and Chronic. The acute response to tissue injury occurs in the microcirculation at the site of injury. First constriction occurs then after the release of chemical mediators causes relaxation of smooth muscles and vasodilation. The Sub-acute phase is characterised by movement of phagocytes to the site of injury. Blood clots are removed by fibrinolysis and damaged tissue are regenerated and replaced with fibroblasts, collagen or endothelial cells. However inflammation becomes Chronic, leading to further destruction and fibrosis. In this the tests compounds have showed maximum inhibition of $48 \%$ at 1 hour of drug treatment at a dose of $10 \mathrm{mg} / \mathrm{kg}$.

\section{Conclusion}

All the five test compounds are tested for anti inflammatory, analgesic and antipyretic activity and found that:

1) All the five compounds are showing anti inflammatory activity but *Bvk.ks.01.16, *Bvk.ks.01.17 and *Bvk.ks.01.18 are showing significant anti inflammatory activity.

2) All the five compounds are showing analgesic activity but Bvk.ks.01.17 and *Bvk.ks.01.18 are showing significant analgesic activity.

3) All the five compounds have no effect on anti pyretic activity.

\section{References}

[1] Albert.Meyers, AikoNabeya, H.WayneAdickes, J. MichealFitzpatrick, G. Ray Malone, and leve R. Politzerpp 1969, Aldehydes from dihydro-1,3-oxazines. II. Synthesis of. Alpha.Beta. -Unsaturated aldehyde and their C-1 deuterated derivatives, J. Am.Chem. Soc 764767.

[2] Basudeb, A; Sukhendu, M.;Dutta, P.K.; Chowdhury,C. 2004 Synette, 14, 2449.

[3] BencsicsS, Elenkov IJ, Vizi EJ. 1995. Alpha 2-, alpha 2A-, alpha $2 \mathrm{~B} / 2 \mathrm{C}$-adrenoceptor subtype antagonist prevent lipopolysaccharideinduced fever response in rabbits. Brain Res; 705:302306.http://dx.doi.org/10.1016/0006-8993(95)01154-4.

[4] B lettes, E.;Lockhart, B.; Lestage, P.; Schwendimann, L.; Gressens,P.; Fleury,, M-B.; Largeron, M.2005 J. Med. Chem. 48,1282

[5] Breder CD, Dinarello CA, Saper CB.1988, Interleukin-1 immunoreactive innervations of the human hypothalamus. Science; 240:321-324.http://dx.doi.org/10.1126/science.3258444.
[6] Buckman, B.O; Mohan, R.; KOovakkat,DS.1998, Bioorg. Med. Chem. Lett.,8, 2235.http://dx.doi.org/10.1016/S0960894X(98)00386-2.

[7] Burke W.J.Am 1954;76;1677.http://dx.doi.org/10.1021/ja01635a065.

[8] C.A Winter, E. Risley, and G. Nuss, "Carrageenan-induced edema in hind aw of the rat as an assay for imflmmatory drugs," Proceedings of the societyfor Experimental Biology and Medicine, vol.111, pp.544-547,1962.http://dx.doi.org/10.3181/00379727-111-27849.

[9] Chiu, H. I.; Lin C.; Cheng, C. Y.; Tsai, M. C.; Yu, H.C. 2001 Bioorg. Med. Chem. Lett., 9, 383.http://dx.doi.org/10.1016/S09680896(00)00260-1.

[10] Chiistie, R.M., Agyako, C.K., Mitchel, 1995 Dyes pigments, 29, 241.http://dx.doi.org/10.1016/0143-7208(95)00049-L.

[11] David,M., Aroff, M.D., Eric, G., Neilson, M.D.,2001. Antipyretics: Mechanism of Action and Clinical Use in Fever Supression.

[12] I. Posadas, M. Bucci, F. Roviezzo et al., "Carrageenan-induced mouse paw oedema is biphasic, age-weight fependant and displays differential nitric oxide cuclooxygenase-2 expression," British journal of pharmacology, vol. 142, no. 2, pp. 331-338, 2004

[13] Jahnke K, thiel E, Bechrakis NE,2008 "Ifosamide or trofosfamide on patients with intraocular lymphoma". J. Neurooncol.93 (2): 213 217.http://dx.doi.org/10.1007/s11060-008-9761-8.

[14] Kajino, N.; Shibouta; Nishikawa, K.; Meguro, K. 1991 Chem. Pharm. Bull. 11 2896.http://dx.doi.org/10.1248/cpb.39.2896.

[15] Katsure, Y.; Nishino, S., Takasugi, H. 1991 Chem. Pharm. Bull. 11, 293.

[16] Li S, Ballou LR, Morham SG.2001 Cyclooxygenase-2 mediates the febrile response of mice to interleukin-1 beta. Brain Res; 910:163173.http://dx.doi.org/10.1016/S0006-8993(01)02707-X.

[17] Li S, Sehic E, Wang Y. 1999 Relationship between complement andthe febrile response of guinea pigs to systemic Endotoxin. Am J Physiol; 277:1635-1645A.

[18] M. Harsh, Inflammation and Healing, Jaypee publication, 2002.

[19] Nair, M.G.; Saltor, O. C.; Kisliuk, R. L.; Gaumont, Y. 1983, J. Med Chem. 26 1164. http://dx.doi.org/10.1021/jm00362a015.

[20] Organic synthesis, Coll Vol. 6, p.905 (1988): Vol. 51, p.24 (1971).

[21] Rawlins, M., 1973. Mechanism of salicylate -induced antpyresis. S Karger AG, 311.

[22] ShaliniDalal, MD, and Donna S. Zhukovsky, MD, FACP2006, Pathophysiology of Fever.

[23] Sharma Sakshy, Hullatti KK. Sachin Kumar, Tiwari KR. Brijesh. Comparative antioxidant activity of Cuscutareflexa and Cassythafiliformis. Journal of Pharmacy Research 2012; 5(1), 441-443.

[24] Sheth UK, Dadkar NK, Kamath NG. Selected topics inexperimental pharmacology. Vol 5, Kothari Book Depot,Bombay, India, 1972, 63.

[25] Shilpkar P. an alternate use of calotropisgigantea: biomethanation. Current science 2007, 92(4); 435-437.

[26] Tripa-

thi KD. Essentials of Medical Pharmacology. 5th Ed. New Delhi: Ja ypee Brothers Medical Publishers; 2003.

[27] Warri-

er, P.K., V.P.K Nambiar, and C. Mankutty 1994. Indian Medicinal Plants. Oriet Longman; Chennai, India p. 341-345.

[28] Vizi ES. 1988, Receptor- mediated local fine-tuning by noradrenergic innervations of neuroendocrine and immune systems. Ann N Y AcadSci; $\quad$ 851: $\quad$ 388-396.http://dx.doi.org/10.1111/j.17496632.1998.tb09012.x.

[29] Watikins LR, Maier SF, Goehler LE. 1995, Cytokine-to-brain communication: a review and analysis of alternative mechanisms. Life Science;57:1011-1026http://dx.doi.org/10.1016/00243205(95)02047-M

[30] Zhou, D. H.; Berger, J.; Clerk, D,; Sethofer, S.G.; Krauss, N. e,;Brothers, J. M.; Martin, TR. S.; Misner, D. L.; Schwabd, D,; Alexandrovad, L.2007, Bioorg. Med. ChemLett. 17, 3504.http://dx.doi.org/10.1016/j.bmcl.2006.12.093. 